\title{
NOTA PRELIMINAR SÔBRE ALGUNS Stomatopoda DA COSTA BRASILEIRA
}

\author{
F. de P. Andrade Ramos
}

Os trabalhos carcinólogos do Instituto Paulista de Oceanografia têm sido conduzidos, com maior intensidade, rumo ao estudo dos Decapoda, especialmente os Macrura, em virtude do papel econômico por êles representado, sobretudo em relação aos camarões de tipo comercial. No entretanto, essa pesquisa, pôsto que em bom andamento, ressente-se de falhas não só devidas à falta de literatura especializada mas, também, decorrentes da impossibilidade de se colecionar material, em tôdas as épocas do ano. À vista disso e em consequência da chegada, ao nosso laboratório, de abundante e variado material carcinológico trazido pelo Diretor do Instituto Paulista de Oceanografia, Snr. Prof. W. Besnard, da excursão por êle empreendida à Ilha da Trindade e a outros pontos da costa brasileira, deliberamos examinar uma parte das coleções dos Arthropoda provenientes dessas regiões. Para início dos estudos, escolhemos a Ordem Stomatopoda, da qual possuimos dois espécimes colhidos por meio do “otter-trawl", no Banco de São Tomé, no litoral do Espírito Santo e quinze de diversos pontos do litoral paulista. Resolvemos, assim, examinar êsses exemplares, apresentando esta nota preliminar sôbre os mesmos.

Estudando as espécies do Pacifico, Schмiтt (1940, p. 139), diz, com muito acerto: "Ainda não conhecemos suficientemente bem os nossos estomatópodos para promover a determinação de espécimes jovens, pequenos ou imaturos". Partindo dêsse princípio, tivemos o euidado de incluir no nosso trabalho sòmente dados sôbre indivíduos bem desenvolvidos. Ainda assim, logo no início, duvidamos da possibilidade de nos encontrarmos em condições de identificar, sem sombra de dúvida, as espécies consideradas por outros autores como frequentes no nosso litoral. Verificamos ainda, que seríamos forçados a adquirir, no exterior, obras fundamentais e indispensáveis aos estudos nomenelaturais, providência que exigiria despesas mais ou menos avultadas ou pelo menos muito superiores ao nível dos nossos recursos financeiros. Recorrendo às bibliotecas especializadas existentes no nosso meio, reconhecemos de pronto que a escassez bibliográfica delimitaria o âmbito das nossas notas sistemáticas, seja quanto à caracterização de alguns gêneros, seja quanto à de certas espécies. Deliberamos, por isso, limitar o nosso campo de ação, apresentando agora uma nota prévia referente às espécies constantes da coleção do Instituto Paulista de Oceanografia. 
É provável que a distribuição geográfica dos espécimes aqui tratados não esteja completa, pela cireunstância de que nem sempre dispusemos de material abundante, bem como de dados exatos quanto aos locais de proveniência do material. Deixaremos, assim, para mais tarde, a tarefa de expender conclusões mais gerais sôbre o assunto.

$\mathrm{Na}$ ordem dos nossos conhecimentos a respeito dos Sstomatópodos da zona litorânea dos continentes meridionais, a América do Sul ocupa, sem dúvida, lugar de muito pequeno destaque. Enquanto o setor do Pacífico já conta com pesquisas substanciais, o Altântico figura entre os mais negligenciados. A zona leste meridional, com o Distrito Federal e os Estados do Rio de Janeiro e Espírito Santo, foi uma das melhores estudadas. Segue-se a região sul, com São Paulo, Paraná, Santa Catarina e Rio Grande do Sul, que rivalisa com o leste setentrional, integrada pela Bahia, Pernambuco e Alagoas. Do nordeste, só conhecemos raras referências ao Cabo $\mathrm{S}$. Roque, afigurando-se-nos nada haver quanto ao extremo norte do país.

Entretanto, os Estomatópodos oferecem campo vastíssimo para pesquisas zoológicas e fisiológicas, não se devendo perder de vista o fato de HANström (1931, p. 200) ter identificado, no pedúneulo ocular de nm representante do gênero Squilla, o órgão " $\mathrm{X}$ " que "pela sua situação e fina estrutura apresentava analogia com os grupos celulares encontrados por Scharrer (1928, p. 32) no mesencefalo de Phoxinus laevis" (SAWAYA 1939, p. 44). Êsse órgão, como se sabe, é considerado como o principal agente na mudança de eôr dos erustáceos.

Com êste subsídio esperamos contribuir, de algum modo, para a investigação de um dos grupos menos conhecidos, no nosso meio, do Phylum do Arthropoda. Na realidade, pouco se sabe ainda a respeito da taxionomia dos exemplares nêle ocorrentes; a maioria dos trabalhos caracterizase por uma pobreza de ilustrações e por tais deficiêneias de diagnose que não só dificultam, sinão até impedem o entendimento das chaves de determinação.

O exame e a classificação dos espécimes colecionados na costa brasileira e constantes das coleções do Instituto Paulista de Oceanografia, só foi possível graças ao auxílio bibliográfico que nos foi gentilmente dispensado por algumas dependências da Universidade de São Paulo e da Secretaria da Agricultura. Somos, por isso, muitos gratos ao Snr. Prof. Dr. Ernesto Mareus, Diretor do Departamento de Zoologia, Dr. Paulo Sawaya, Diretor do Departamento de Fisiologia e Dr. Oliverio Mario de Oliveira Pinto, Diretor do Departamento de Zoologia, da Secretaria da Agricultura, pelo acesso franco às bibliotecas dos citados Departamentos.

Cumpre-nos ainda agradecer ao Snr. Prof. W. Besnard, Diretor do Instituto Paulista de Oceanografia, a coleta do material obtido durante a excursão à Ilha da Trindade, bem como à Da. Hilda M. Texeira e Silva, pela oferta do material proveniente do Mereado Municipal de S. Paulo e ao Snr. Clarimundo de Jesus, pela obtensão dos espécimes de Santos. Agradecemos, também ao Snr. Kossako Kikuchi, armador, residente em 
Santos, a remessa da maioria dos exemplares que figuram neste trabalho. Ao Snr. J. de Paiva Carvalho, Chefe da Seção da Fauna Marítima, agradecemos a orientação imprimida ao presente trabalho e a confecção dos desenhos que nêle figuram.

Os Estomatópodos não escaparam à arguta observação de MARCGRAve que, ao se referir à fauna earcinológica do Brasil, não deixou de mencionar a "Tamarutáca" ou "Tamburutáca", dela fornecendo desenho muito fiel.

Uma das alusões mais antigas aos representantes das nossas águas é a de Brooks (1886), que tratou de Lysiosquilla (Lisioerichthus) triangulares, do Rio de Janeiro.

Infelizmente, no nosso meio, êsses crustáceos têm sido muito pouco estudados. Inering (1897, p. 156), ao apreciar a fauna da Ilha de São Sebastião, no Estado de São Paulo, diz: "Outra espécie parecida com os camarões é a lagosta-gafanhoto (Squilla scabricauda Latr.,) que se assemelha ao gafanhoto louvadeus e que vive no lôdo, servindo aos pescadores de isca'". Moreira (1901, p. 1-5) relaciona as seguintes espécies: Squilla dubia M. Edwards, S. prasinolineata Dana, Lysiosquilla scabricauda (Lam.,) e Gonodactylus falcatus (Forskael). MoreIRA (1905, p. 157) assinala duas espécies que teve a oportunidade de estudar durante as eampanhas do Annie; Chlorodiella empusa (Say) = Squilla empusa Say e Hemisquilla braziliensis (Moreira) = Pseudosquilla braziliensis Moreira. Relacionando as espécies da costa bandeirante, LUEDERwaLdT (1919, p. 429), inclúe Lysiosquilla scabricauda (Lam.,) de Ubatuba e Ilha de São Sebastião e Chlorodiella dubia Milne-Edwards, de Santos, Iguape, São Sebastião e Ilha de São Sebastião. Aliás, dez anos mais tarde, êsse mesmo autor (1929, p. 52), reafirma a existência dessas duas espécies no litoral paulista.

Na relação dos erustáceos capturados na Ilha da Trindade, Moreira (1920, p. 130-132), não faz nenhuma menção a respeito da ocorrência de Stomatópoda na região. Os dados que possuímos sôbre outros pontos da costa do Brasil são relativamente escassos. Oliveira (1940, p. 145), dá como existentes na baía de Guanabara as seguintes espécies: Lysiosquilla scabricauda, Lysiosquilla (Lysioerichthus triangularis, Chlorodiella dubia e Squilla prasinolineata. Cineo anos mais tarde, o mesmo autor (1945, p. 335-337), faz referência ao grupo, desta vez, porém, para deserever a espécie larvária Squillerichtus aragaoi, da enseada de Jurujuba.

Até o presente, diversos autores assinalaram 5 gêneros e 9 espécies em águas brasileiras, a saber:

I - Gênero Squilla Fabricius, 1787.

1 - Squilla empusa Say, encontrada entre 15 e 20 milhas da costa do Distrito Federal, na altura da Ilha Rasa, em profun- 
didade de 60 a 80 metros; na Ilha Grande, entre o ilhóte Pau a Pino e as enseadas das Palmas e do Céu, na profundidade de 30 metros; na Ponta de Guaratiba, a 24 metros de profundidade (Moreira, 1905).

2 - Squilla prasinolineata Dana Mrers (1880, p. 4), assinala a espécie, sem mencionar a localidade; mais adiante, porém p. 19-20), esclarece que o exemplar manipulado por Dana provinha do Rio de Janeiro. Moreira (1903, p. 2) São Franciseo do Sul (Estado de Santa Catarina). Baía de Guanabara (OLIverra 1940).

3 - Squilla dubia Milne - Edwards (=Chlorodiella dubia). Mrers (1. c. p. 24), dá como distribuição geográfica da espécie: "Costa oriental da América do Norte, América Central e do Sul". Santos, Iguape, São Sebastião, no Estado de São Paulo (Luederwaldt 1919, p. 429; 1929, p. 52). Mereado Municipal de São Paulo, proveniente do litoral paulista (sem data). Dádiva da Exma. Snra. Da. Hilda M. Texeira e Silva.

4 - Squilla brasiliensis Calman. Cabo Frio (Calman, 1917); Sснмiтt 1940, p. 167).

II - Gênero Lysiosquilla Dana, 1852.

5 - Lysiosquilla scabricauda (Lamarek). Mrers (l. c., p. 8) menciona a ocorrência da espécie na região atlântica, acrescentando que "os espécimes do Museu Britânico são provenientes do Brasil'". Costas americanas do Oceano Atlântico, desde Charleston (GIBBES), até São Francisco, no Estado de Santa Catarina, Brasil (Moreira 1901, p. 1). Ubatuba e São Sebastião, Estado de São Paulo (LuEderWALDT 1919 , p. $429 ; 1929$, p. 52). Baía de Guanabara (Oliveira 1940, p. 145). Catorze exemplares, provenientes de Santos, fazem parte das coleções do Instituto Paulista de Oceanografia.

III - Gênero Hemisquilla Hansen, 1895.

6 - Hemisquilla braziliensis (Moreira). Proximidades da Ilha Rasa a 30 milhas da costa, em profundidade de 80 a 100 metros; circunvizinhanças da Ilha do Jorge Grego, a SE da Ilha Grande, em profundidade de 80 metros (MoreIra 1903): Banco de São Tomé, no litoral do Espírito Santo, em profundidade de 24 metros, coletado, em maio de 1950, pelo Prof. W. Besnard. 
IV - Gênero Pseudosquilla Dana, 1852.

7 - Pseudosquilla oculata (Brullé). Maceió, Alagoas (Schмiтt 1940, p. 174).

V - Gênero Gonodactylus Latreille, 1825.

8 - Gonodactylus oerstedii Hensen. Brasil Fernando de Noronha (Schmitт 1940, p. 211). Não se trata, evidentemente, da fórma típica que Bigelow (1931, p. 122) diz ser "amplamente repandida nas águas superficiais costeiras do continente e ilhas atlânticas da América tropical, como foi indicado por Sмrтн (1890), que examinou espécimes das Bermudas Florida Keys, Aspinwall (agora Colon), Caravelas e Abrolhos, no Brasil'. Conquanto o citado autor tenha dito que "Pocock (1890) assinalou a presença desta espécie em Fernando de Noronha, no Atlântico Sul", somos propensos a erer que não se trata da fórma típica, mas sim da que possue telson earacterístico do Atlântico (Sснмiтt, 1. e., p. 213, fig. 29, $a$ e $b$ ).

9 - Gonodactylus oerstedii var. spinulosus Schmitt. Cabo de São Roque (Schмiтt 1924, p. 96-98).

Neste trabalho, não nos ocuparemos das fórmas larvárias - Lysiosquilla (Lysioerichtus) triangularis, nem de Squillerichtus aragaoi (OLIveIRA) 1940 , p. $145 ; 1945$ p. 335-337). Deixamos também de lado três exemplares de gênero Gonodactylus. $\mathrm{O}$ assunto será, provàvelmente, abordado mais tarde, à luz das ehaves analíticas de Bigelow (1894, p. 543-549), depois que se tiver promovido o exame do material planetônico proveniente da excursão à Ilha da Trindade.

Em águas nacionais, há ainda a possibilidade de se encontrar Lysiosquilla scolopendra (Latreille), que Mrers (1. c., p. 3) supõe frequentar a costa brasileira, dizendo que ela "faz parte da coleção de Crustáceos trazida do Brasil por M. Lalande"'. Morerra (1901, p. 2) diz, a êsse respeito: "Claus dá Lysiosquilla scolopendra (Ltr.), Coronis scolopendra Ltr., como do Brasil, (Grundzuger der Zoologie v. I, p. 610 (1880); esta asserção é, entretanto, se não infundada, ao menos problemática, pois nem M. Edwards (Hist. Nat. Crust., v. II, p. 53 (1837), nem Miers (Ann. and Mag. of Nat. Hist. (5) V, p. 9 (1880) garantem-lhe essa procedência, inclinando-se antes a crer que Lalande a tenha obtido na ilha da Madeira e reunido a outros Crustáceos no Brasil'’. Kemp (1913, p. 204), menciona, com reservas, a ocorrência dessa espécie no Brasil. Este autor (l. c., p. 201) faz figurar, no nosso território, Squilla panamensis Bigelow, mas é provável que se trate de Squilla brasiliensis Calman que, tanto êle como Sснмiтt (1. e., p. 167) admitem ser semelhante à "Variedade C", da espécie de Bigelow. Quanto à Gonodactylus falcatus 
(Forskael), Moreira (1901, p. 1), diz que S. J. Smith põe “em dúvida a identidade dos exemplares coligidos pelo Prof. Hartt em Abrolhos e Caravellas, Estado da Bahia - com o G. falcatus do antigo continente, reconhece, entretanto, que não diferem da espécie própria das Antilhas e Flórida, que B. Sharp considera como G. falcatus e, segundo êste naturalista, vive em todos os mares". Essa opinião, aliás, já fôra esposada por Stebing (1893, p. 286-287).

A determinação dos Stomatópoda das coleções do Instituto Paulista de Oceanografia foi baseada na chave de determinação dos gêneros organizada por Kemp (1. c., p. 16) e modificada por SchmitT (1. c., p. 137-138, como segue):

I - Articulação ísquio-humeral da garra preensora terminal normal; merus com a porção inferior entalhada, de modo a abrigar o propodus em tôda a sua extensão; propodus finamente pectinado ou com uma série de espinhos fixos ao longo da margem externa da superfície dorsal; dactylus sem entumescimento na base, salvo quanto ao gênero Coronida.

A - Carapaça dotada de carenas evidentes; sulco cervical bem pronunciado, ocupando todo o dorso; primeiros cinco somitos abdominais com carenas longitudinais; dactylus da garra preensora armado de dentes na margem interna ... Squilla.

B - Carapaça sem carena (exceto em Pseudosquilla ferrusaci Roux, do Mediterrâneo); sulco cervical ausente no dorso da carapaça; ausência de carenas do primeiro ao quinto somito abdominal.

(1) Abdomen geralmente comprimido, raramente muito achatado (como em Pseudosquilla valeronis); telson com carena mediana bem evidente, profunda e (nos adultos), geralmente acompanhada de uma ou mais carenas adicionais de cada lado; dentes submedianos com pontas ou espinhos móveis; ausência de dentículos submedianos (ao menos nos adultos).

a) Dactylus da garra preensora, com 3 a 4 dentes na margem interna, mas, às vêzes, existindo de 7 a $9(P$. valeronis), incluindo nesse número o terminal ... Pseudosquilla.

b) Dactylus da garra preensora sem dentes na margem interna, salvo o terminal...$\ldots \ldots \ldots \ldots$. Hemisquilla

(2) Abdomen achatado dorso-ventralmente.

a) Rostro com espinhos mediano interno; dactylus da garra preensora sem entumescimento na base, armado, ao menos, com 4 dentes (sem contar o terminal), na margem interna; telson sem carena mediana verdadeira, liso ou rugoso, sem espinhos ou dentículos submedianos ou com fileira transversa ou anel de espinhos .... Lysiosquilla. 
b) Dactylus da garra preensora com a base entumescida, provido de 3 dentes (não incluindo o terminal); telson engrossado, fortemente guarnecido de espinhos pequenos, espínculas delicadas ou tubérculos grandes; presença ou ausência de carenas submedianas ......... Coronida.

aa) Rostro anteriormente dividido por incisão profunda, formando dois prolongamentos fortemente denteados

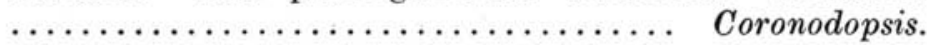

II - Articulação isquio-humeral da garra preensora não terminal, situada um tanto anteriormente, em relação à extremidade proximal do merus; dêsse ponto, na direção dorsal, essa articulação se torna mais evidente; superfície ventral do merus escavada, local onde o propodus se abriga em $3 / 4$ partes do seu comprimento; dactylus com a base entumescida.

a) Dactylus da garra preensora sem dentes na margem interna, salvo o terminal ....................... Gonodactylus.

b) Dactylus da garra preensora com dentes na margem interna, além do terminal ................... Odontodactylus.

Gênero Squilla Fabricius, 1787.

Squilla brasiliensis Calman

Est. I, fig. 1.

Squilla panamensis Bigelow 1894, vol. 17, p. 526, fig. 17-18; Kemp 1913, vol. 4, p. 201; Schmitt 1940, p. 167.

No estudo dos exemplares pertencentes ao gênero Squilla, seguimos Schмiтt (l. c., p. 139), isto é, consideramos a prioridade dêsse gênero sôbre Clorida Eydoux e Souleyet e sôbre Chloridella Miers.

Na diagnose desta espécie da baía de Paranaguá (Estado de São Paulo) e de Peruíbe (Estado de São Paulo), consideramos os seguintes caractéres: olhos bilobados; cinco primeiros segmentos abdominais providos de carenas longitudinais; dactylus da garra preensora não entumescido na base, com 4 dentes na margem interna; telson unido ao sexto segmento abdominal por meio de articulação móvel; porção posterior do corpo, larga e deprimida; presença de 3 dentículos intermediários e 2 submedianos, no telson; lóbulos pre-laterais pequenos, não terminando em ponta; em um dos exemplares, o dente lateral esquerdo do telson acha-se fundido com o intermediário (fig. 1) ; carena mediana terminando em ponta fina; na porção anterior da carena há uma fissura transversa bem pronunciada. 


\begin{tabular}{|c|c|c|}
\hline 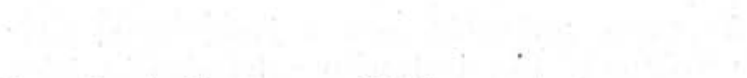 & $\begin{array}{c}\text { Exemplar } \\
\text { N.. } 1\end{array}$ & $\begin{array}{c}\text { Exemplar } \\
\text { N.o } 2\end{array}$ \\
\hline Comprimento do corpo, exeluindo o rostro ........... & $145 \mathrm{~mm}$ & $116 \mathrm{~mm}$ \\
\hline 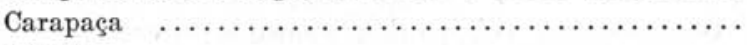 & $39 "$ & $26 "$ \\
\hline Rostro $\ldots \ldots \ldots \ldots \ldots \ldots \ldots \ldots \ldots$ & 6 & $"$ \\
\hline Largura anterior da earapaça $\ldots \ldots \ldots \ldots \ldots \ldots \ldots$ & $15 "$ & $14 "$ \\
\hline Proporção da earapaça no comprimento total ..... & $1 / 11$ & $1 / 8$ \\
\hline
\end{tabular}

A espécie é muito semelhante à "Variedade C", de S. panamensis Bigelow, conforme acentuaram Calman e Schmitt.

Ocorrência: Baía de Paranaguá (Estado do Paraná); Peruíbe (Estado de São Paulo); Cabo Frio, onde Calman (l. c., p. 139) obteve o tipo.

Squilla dubia H. Milne - Edwards

Est. I, fig. 2.

Squilla alba Boone 1930, vol. 2, p. 35; Squilla dubia Biglow 1937, vol. 17, p. 518; Schmitt 1940, vol. 5, n. ${ }^{\circ} 4$, p. $155-156$.

O pedúnculo ocular dilatado, em forma de garrafa, com estrangulamento distal logo abaixo da córnea disposta oblìquamente sôbre o pedúnculo ocular identifica perfeitamente esta espécie do litoral paulista.

A garra acha-se provida de 6 dentes, cinco grandes e um muito pequeno. A carena mediana não é bifureada; os processos laterais do $5 .^{\circ}$ sômito toráxico possuem um espinho pontudo de cada lado, ligeiramente inclinado para a frente. A fórmula dos dentículos é a seguinte: $2,3,1$.

Comprimento do corpo excluindo o rostro, $120 \mathrm{~mm}$; carapaça $25 \mathrm{~mm}$; rostro $4 \mathrm{~mm}$; largura anterior da earapaça $13 \mathrm{~mm}$; proporȩão da carapaça no comprimento total $1 / 9$.

Ocorrência: Adquirido no Mercado Municipal de São Paulo e ofertado gentilmente às coleções do Instituto Paulista de Oceanografia, pela Exma. Snra. Da. Hilda M. Texeira e Silva.

Gênero Lysiosquilla Dana, 1852.

Lysiosquilla scabricauda (Lamarek)

Est. I, fig. 3.

Lysiosquilla scabricauda MIERs 1880, p. 8; MoReIRA 1901, p. 1-2; LUEDERWALDT 1919 , p. 429; 1929, p. 52; Oliveira 1940, p. 145.

O corpo anterior é mais ou menos achatado e desprovido de carenas longitudinais; corpo posterior alargado e deprimido; sexto segmento 
abdominal separado do telson por articulação móvel; olhos grandes e triangulares; dactylus da garra preensora com 9 dentes fortes, não tendo entumescimento na base; telson de formato semicircular, rugoso, amplo, mais largo do que comprido, dotado de granulações finas de cada lado da carena mediana, em forma de língua; faces laterais e pre-laterais e intermediários curvados para dentro, os segundos laterais providos de dois pequenos prolongamentos; dentes submedianos (um de cada lado), pequenos e quase retos; dentículos submedianos, em números de 4 de eada lado, fundidos e com a margem posterior sinuosa. Êsse curioso tipo de telson, segundo Berry (1939, p. 462) foi comparado com o de Squilla antiqua, por MÜNSTER (1842), que estudou êste espécime fóssil do Eoceno.

Moreira (1901, p. 2) diz que "segundo Miers, a espécie descrita por Herklots como Squilla Haeveni e procedente da costa ocidental africana é idêntica a esta, o que vem provar que sua área de dispersão geográfica se estende a tôdas as costas americanas e africanas de iguais latitudes, banhadas pelo Atlântico"'.

As medidas obtidas em 14 (catorze) exemplares, são as seguintes:

\begin{tabular}{|c|c|c|c|c|c|c|c|c|c|c|c|c|c|c|}
\hline & \multicolumn{14}{|c|}{$\begin{array}{c}\text { E X E M P L A R } \\
\text { N. }{ }^{\circ}\end{array}$} \\
\hline & \multicolumn{14}{|c|}{ Medidas em milímetros } \\
\hline & 1 & 2 & 3 & 4 & 5 & 6 & 7 & 81 & 9 & 10 & 11 & 12 & $|13|$ & 14 \\
\hline $\begin{array}{l}\text { Comprimento } \\
\text { do corpo sem } \\
\text { o rostro...... }\end{array}$ & 160 & 171 & 171 & 172 & 187 & | 198 & |207 & $210 \mid$ & $|222|$ & 242 & 175 & $\mid 224$ & 180 & 160 \\
\hline Carapaça .... & 30 & 32 & 33 & 34 & 35 & 40 & 39 & 34 & 44 & $|43|$ & 34 & $42 \mid$ & $|34|$ & 29 \\
\hline Rostro....... & 6.5 & 7 & 8 & 9 & 7 & 9 & 9 & 9 & 9 & 9 & 8 & 9 & 8 & \\
\hline $\begin{array}{l}\text { Largura ante- } \\
\text { rior da cara- } \\
\text { paça....... } \\
\end{array}$ & 30 & 36 & 30 & 36 & 40 & 37 & 45 & 28 & $4 \varepsilon$ & 30 & 27 & 43 & 32 & 33 \\
\hline $\begin{array}{l}\text { Carapaça no. } \\
\text { comprimento } \\
\text { total .......... }\end{array}$ & $\begin{array}{c}1 \\
5.3\end{array}$ & $\mid \begin{array}{c}1 \\
5.7\end{array}$ & $\begin{array}{c}1 \\
4.7\end{array}$ & $\left|\begin{array}{c}1 \\
4.8\end{array}\right|$ & $\begin{array}{c}1 \\
4.7\end{array}$ & $\begin{array}{l}1 \\
5\end{array}$ & $\begin{array}{c}1 \\
4.6\end{array}$ & $\begin{array}{l}1 \\
5\end{array}$ & $\begin{array}{c}1 \\
5.7\end{array}$ & $\begin{array}{l}1 \\
8\end{array}$ & $\left|\begin{array}{c}1 \\
5.1\end{array}\right|$ & $\left|\begin{array}{c}1 \\
5.3\end{array}\right|$ & $\left|\begin{array}{c}1 \\
5.2\end{array}\right|$ & $\begin{array}{c}1 \\
5.5\end{array}$ \\
\hline
\end{tabular}
arrastão.

Os espécimes de Santos foram capturados, à noite, por meio do

Ocorrência: Santos, São Sebastião, Ubatuba, Baía de Guanabara, São Franciseo (Estado de Santa Catarina). 
Gênero Hemisquilla Hansen, 1895

Hemisquilla braziliensis (Moreira)

Est. I, fig. 4.

Sсhмiтt 1940 , p. 180 , fig. 18 b.

O corpo anterior é achatado, com o sexto segmento abdominal não fundido com o telson; corpo posterior liso e muito convexo; o rostro é triangular não sendo iniciado por ponta aguda; os olhos são claviformes ; o dactylus da garra preensora não possue nenhum dente; o telson (fig. 3) tem, de largura, o dôbro do comprimento, achando-se munido de seis dentes marginais; dentes pre-laterais, recurvados para dentro, mas sem espinho apical; dentes submedianos providos de espinho apical; nos locais onde deviam figurar os dentículos intermediários, há uma excreeência que termina com espinho aguçado; sôbre o telson, figuram o tubéreulo mediano que é estreito e alongado e dois outros laterais, quase crescentiformes.

\begin{tabular}{|c|c|c|}
\hline & $\begin{array}{c}\text { Exemplar } \\
\text { N.० } 1\end{array}$ & $\begin{array}{c}\text { Exemplar } \\
\text { N. }{ }^{\circ} 2\end{array}$ \\
\hline Comprimento do corpo, exeluindo o rostro $\ldots \ldots \ldots \ldots$ & $176 \mathrm{~cm}$ & $123 \mathrm{~cm}$ \\
\hline Carapaça & $33 ”$ & $24 ”$ \\
\hline 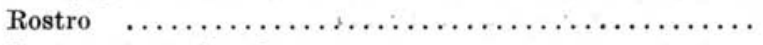 & $8 ”$ & \\
\hline Largura anterior da carapaça $\ldots \ldots \ldots \ldots \ldots \ldots \ldots$ & $25 "$ & $18 "$ \\
\hline Carapaça contida no comprimento total $\ldots \ldots \ldots \ldots \ldots$ & $1 / 7$ & $1 / 7$ \\
\hline
\end{tabular}

Ocorrência: Ilha Rasa, Ilha do Jorge Grego e Banco de São Tomé.

\section{SUMÁRIO}

Tendo o Instituto Paulista de Oceanografia recebido abundante material carcinológico coletado pelo seu Diretor, Prof. W. Besnard, na excursão realizada à Ilha da Trindade e a outros pontos do País, deliberou o autor examinar os Arthropoda dessa região.

Conquanto interessado, particularmente, pelos Decapoda Macrura, deliberou o autor estudar os Stomatopoda recebidos, desde que, além dêles, as coleções do Instituto Paulista de Oceanografia possuíam diversos representantes do litoral do Estado de São Paulo e da costa do Estado do Espírito Santo.

$\mathrm{Na}$ ordem dos nossos conhecimentos a respeito dos exemplares da região litorânea dos continentes meridionais, a América do Sul ocupa, sem dúvida, lugar de muito pequeno destaque. Enquanto o setôr do Pacífico já conta com pesquisas substanciais nesse terreno, o Atlântico figura entre as regiões mais negligenciadas. 
Nesta nota prévia, examinam-se, portanto, 9 (nove) espécies assinaladas por diversos autores, ao longo da costa brasileira. Delas, até o presente, foram identificadas, nas coleções do Instituto Paulista de Oceanografia, 4 (quatro). $\mathrm{O}$ autor se manifesta sôbre as mesmas, dando de cada uma um desenho elucidativo de suas partes essenciais, de modo a facilitar a outrem a identificação. Dessa maneira, espera o autor ter contribuido, de algum modo, para a investigação de um dos grupos do Phylum dos Arthopoda, menos pesquisados no Brasil.

\section{ABSTRACT}

As the Instituto Paulista de Oceanografia (The São Paulo Oceanographic Institute) received a great deal of carcinological material collected by its Director, Prof. W. Besnard, on the trip made to the Ilha da Trindade $\left(20^{\circ} 30^{\prime} 00^{\prime \prime} \mathrm{S}\right.$. \& $29^{\circ} 22^{\prime} 00^{\prime \prime} \mathrm{W}$.), the author resolved to examine the Arthropoda of that region.

Although especially interested in the Decapoda Macrura, the author determined to study the received Stomatopoda, considering that, besides these samples, the collections of the Instituto Paulista de Oceanografia possessed several specimens of the littoral of the State of S. Paulo and of the coast of the State of Espirito Santo.

Respecting our knowledge about the specimens of the littoral region, South America surely occupy but a place of little importance in the range shown substancial researches in that matter, the Atlantic region is considered as one of the most neglected.

In this previous note we are considering, therefore, nine species found by several authors along the Brazilian coast. From these ones, four could be identified in the collections of the Institute. The author refers to them, giving an explaining sketch of the essential parts of each of them. Thus, the author hopes to have given a contribution, at this rate, to the investigation of one of the groups of the Arthropoda Phylum, less studied in Brazil.

\section{BIBLIOGRAFIA}

BERRY, C. T., 1939 - A summary of the fossil Crustacea of the Order Stomatopoda and deseription of a new species from Angola. The Amer. Midd. Nat., vol. 21, n. ${ }^{\circ}$ 2, p. 461-471. Indiana.

BIGELOW, R. P., 1894 - Seientific Results of explorations by the U. S. Fish Comission Albatross, XXXII. Report on the erustacea fo the order Stomatopoda coll. by steamer Albatross, between 1885 and 1891, and other specimens in the U. S. Nat. Mus., vol. XVII, p. 489-550. Washington.

BIGELOW, R. P., 1931 - Stomatopoda of the southern and eastern Pacific Ocean and Hawaiian Islands. Bull. Mus. Comp. Zool., vol. LXXII, n. ${ }^{4}$ 4, p. 105-191, est. I-II. Cambridge. 
BOONE, L., 1930 - Crustacea: Stomatopoda and Brachyura. Scientific Results of the eruises of the yachts "Eagle" and "Ara", 1921-1928. William Vanderbilt commanding. Bull. of the Vanderbilt Marine Museum, vol. II, p. 1-228. New York.

BROOKS, W. K., 1886 - Report on the Stomatopoda collected by H. M. S. Challenger, Zool., vol. XVI, p. 1-116, est. I-XVI. London.

HANSTRÖM, B., 1931 - Neue Untersuchungen über Sinnesorgane und Nervensystem der Crustaceen. I. Zeit. Morph. u. Ökolog. Tiere, vol. 23, p. 80-236. Berlin.

IHERING, H. von 1897 - A Ilha de São Sebastião. Rev. Mus. Paulista, vol. II, p. 129-171. São Paulo.

KEMP, S., 1913 - An account of the Crustacea Stomatopoda of the Indo-Paeifie region based on the collection in the Indian Museum. Mem. Ind. Mus., vol. IV, n. ${ }^{\circ}$, p. 1-127, est. I-X. Caleutá.

LUEDERWALDT, H., 1919 - Lista de Crustáceos superiores (Thoracostraca) do Museu Paulista, que foram encontrados no Estado de São Paulo, por H. Luederwaldt, naturalista do Museu Paulista. Rev. Mus. Paulista, vol. XI, p. 429-435. São Paulo.

LUEDERWALDT, H., 1929 - Resultados de uma excursão scientifica à Ilha de São Sebastião, no litoral do Estado de São Paulo e em 1925. Rev. Mus. Paulista, vol. XVI, p. 1-79. São Paulo.

LUNZ, G. R., jr., 1937 - Stomatopoda of the Bingham Oceanographic Collection. Bing. Oc., Coll. vol. V, art 5, p. 1-19, 10 figs. New Haven.

MIERS, E. J., 1880 - On the Squillidae. Ann. \& Mag. Nat. Hist., ser. 5, vol. V, p. 1-30. London.

MOREIRA, C., 1901 - Contribuição para o conhecimento da fauna brasileira. Crustáeeos do Brasil. Areh. Mus. Nae. do Rio de Janeiro, vol. XI, p. 1-151, Rio de Janeiro.

MOREIRA, C., 1903 - Crustáceos da Ponta do Pharol em S. Franciseo do Sul, no Estado de Santa Catarina. Arch. Mus. Nac. do Rio de Janeiro, v. XII, p. 119-123. Rio de Janeiro.

MOREIRA, C., 1905 - Campanhas de Pesea do "ANNIE". Crustáceos. Areh. Mus. Nae. do Rio de Janeiro, vol. XIII, p. 124-145, est. I. Rio de Janeiro.

MOREIRA, C., 1920 - Faune Careinologique de L'lle de la Trindade. Bull. Soc. Zool. France, vol. XLV, n. ${ }^{\circ}$ 3-7, p. 125-132. Paris.

OLIVEIRA, L. P. H., 1940 - Contribuição ao conhecimento dos erustáceos do Rio de Janeiro. Catálogo dos erustáceos da Baía de Guanabara. Mem. Inst. Oswaldo Cruz, vol. 35, fase. 1, p. 137-151. Rio de Janeiro.

OLIVEIRA, L. P. H., 1945 - Sôbre uma nova espécie de Crustáceo Stomatopoda, Squillerichtus aragaoi. Mem. Inst. Oswaldo Cruz, vol. 41, fase. 2, p. 335-336, 1 est. Rio de Janeiro.

SAWAYA, P., 1939 - Sôbre a mudanȩa de eôr nos Crustáceos. Contribuição para o estudo da fisiologia dos cromatóforos e dos hormônios dos invertebrados. Tese, p. 1-106. São Paulo.

SCHMITT, L. W. 1940 - The Stomatopods of the west coast of Ameriea. Allan Hancock Pacifie Expedition, vol. 5, n. ${ }^{\circ} 4$, p. 127-225. Los Angeles.

STEBING, T. R. R., 1893 - A History of Crustacea. The International Scientific Series, XVII+466, D. Appleton \& Company. New York. 


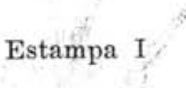

\section{EXPLICAÇÃO DA ESTAMPA I}

Fig. 1 Squilla braziliensis Calman, de Peruíbe, Estado de São Paulo.

Fig. 2 Squilla dubia H. Milne-Edwards. Mereado Municipal de São Paulo.

Fig. 3 Lysiosquilla scabricauda (Lamarek), de Santos, Estado de São Paulo.

Fig. 4 Hemisquilla braziliensis (Moreira), do Banco de São Tomé, E. do Espírito Santo. 


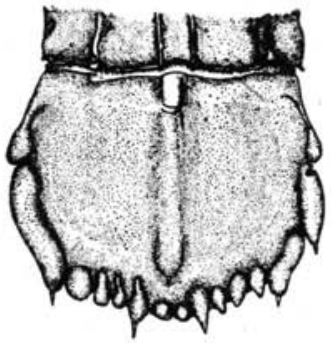

FIG. 1

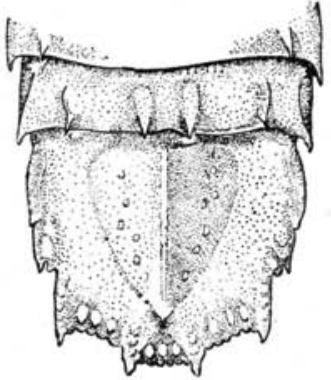

FIG. 2

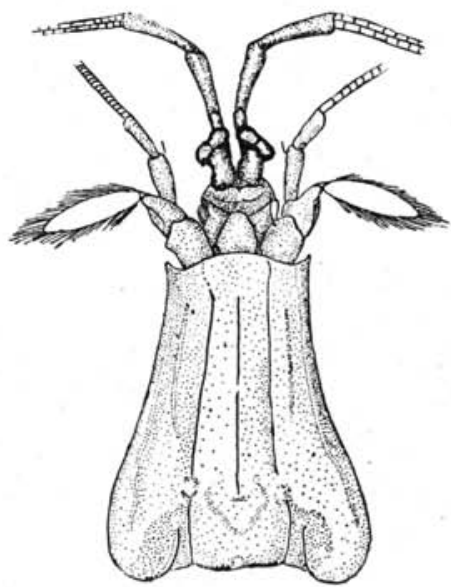

FIG. 2
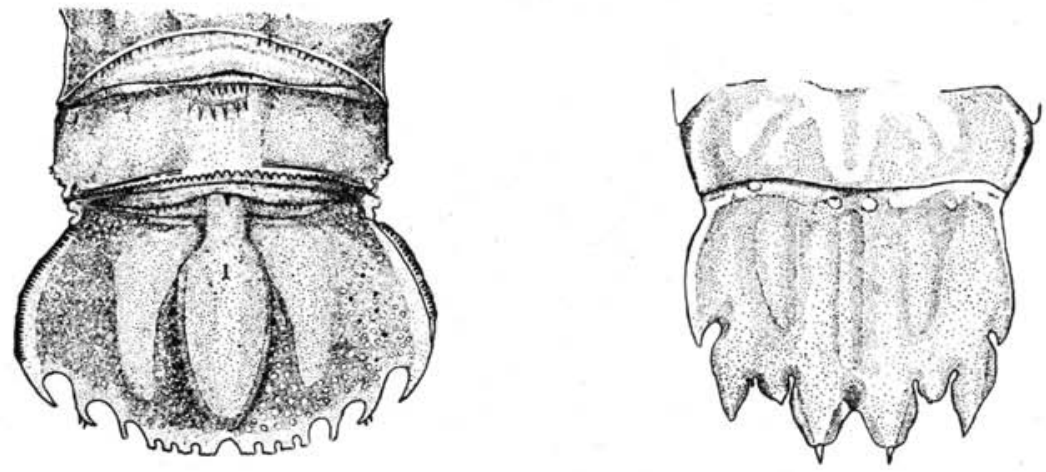

FIG. 3

FIG. 4 